\title{
ROOM TEMPERATURE LASER ACTION IN LATERALLY OVERGROWN GaN PYRAMIDS ON (111) SILICON
}

\author{
S. Bidnyk,* B. D. Little,* Y. H. Cho,* J. Krasinski,* J. J. Song,* \\ W. Yang, ${ }^{* *}$ and S. A. McPherson** \\ *Center for Laser and Photonics Research and Department of Physics, \\ Oklahoma State University, Stillwater, Oklahoma 74078 \\ **Honeywell Technology Center, Plymouth, Minnesota 55441
}

Cite this article as: MRS Internet J. Nitride Semicond. Res. 4S1, G6.48 (1999)

\begin{abstract}
Single and multi-mode room temperature laser action was observed in GaN pyramids under strong optical pumping. The 5- and 15-micron-wide hexagonal-based pyramids were laterally overgrown on a patterned GaN/AlN seeding layer grown on a (111) silicon substrate by metal-organic chemical vapor deposition. The pyramids were individually pumped, imaged, and spectrally analyzed through a high magnification optical system using a high density pulsed excitation source. We suggest that the cavity formed in a pyramid is of a ring type, formed by total internal reflections of light off the pyramids' surfaces. The mode spacing of the laser emission was found to be correlated to the size of pyramids. The effects of pyramid geometry and pulse excitation on the nature of laser oscillations inside of the pyramids is discussed. Practical applications of the results for the development of light-emitting pixels and laser arrays are suggested.
\end{abstract}

\section{INTRODUCTION}

$\mathrm{GaN}$ and GaN-based heterostructures have been extensively studied due to their potential applications in the UV-blue spectral region ${ }^{1}$ in a wide temperature range..$^{2-4}$ The success in fabrication of long lifetime cw edge-emitting laser diodes (LDs) was largely due to a significant reduction in defects attained by using laterally grown $\mathrm{GaN}$ on sapphire substrates. ${ }^{5}$ The selective growth of wide bandgap semiconductors is believed to be one of the most important methods to realize high performance LDs in the short wavelength region. ${ }^{6,7}$ However, because of the large physical dimensions of their resonator cavities (several hundred microns), traditional edgeemitting lasers may only be used for constructing one-dimensional arrays. On the other hand, surface-emitting lasers or microstructure-based lasers ${ }^{8}$ (with a typical cavity of a few microns) could potentially be used for the development of two-dimensional laser arrays. In this work we demonstrate single-mode and multi-mode room temperature (RT) laser action in twodimensional arrays of hexagonal GaN pyramids grown on (111) Si by the selective lateral overgrowth technique. We show that the geometry of each pyramid allows the formation of a high-finesse cavity through the utilization of total internal reflection inside of the pyramids.

\section{EXPERIMENT}

Samples used in this study were grown by low-pressure metal-organic chemical vapor deposition (MOCVD). First, a 0.10- $\mu \mathrm{m}$-thick AlN buffer layer was deposited on the Si wafer and the GaN layer was subsequently grown resulting in a thickness of about $0.15 \mu \mathrm{m}$. To prepare samples for selective growth, a $0.1-\mu \mathrm{m}$-thick $\mathrm{Si}_{3} \mathrm{~N}_{4}$ masking layer was deposited on the wafer by 
plasma-enhanced chemical vapor deposition. Arrays of openings were created by photolithography and reactive ion etching. The openings were arranged in a hexagonal pattern with a $20 \mu \mathrm{m}$ spacing and the average diameter of the openings ranged from 2 to $5 \mu \mathrm{m}$ depending on the size of pyramids to be grown. GaN growth was then performed in the MOCVD reactor with the ammonia flow set at $1.8 \mathrm{slm}$ and the triethylgallium (TEG) flow varying 1.9 to $5.3 \mu \mathrm{mol} / \mathrm{min}$ over the course of 3 hours at a temperature of $1050{ }^{\circ} \mathrm{C}$. The smaller TEG flow for the initial growth was used to avoid nucleation on the mask. The growth conditions are described elsewhere ${ }^{9}$ in more detail. The result of the selective lateral overgrowth was a two-dimensional array of GaN pyramids. A scanning microscope image (SEM) image of one of the samples is shown in Fig. 1. The base diameter of the pyramids was estimated to be about 5 and $15 \mu \mathrm{m}$ for the two different arrays of pyramids, which is considerably larger than corresponding 2 and $5 \mu \mathrm{m}$ openings in the mask, indicating substantial lateral growth of the pyramids. Transmission electron microscope pictures revealed a drastic reduction in defect densities.

The samples were mounted on a translation stage that allowed 3-D positioning of the sample with $\sim 1$ micron resolution. The third harmonic of an injection-seeded Nd:YAG laser was used as the pumping source. The pulse width of the laser was varied from 5 to $25 \mathrm{~ns}$ by changing the Q-switch delay. The laser beam was focused to a diameter of $4 \mu \mathrm{m}$ through a microscope objective. The laser light intensity was attenuated continuously using a variable neutral density filter. This study was performed in a surface emitting geometry where emission from the sample was collected through the same microscope objective in the direction normal to the sample surface.

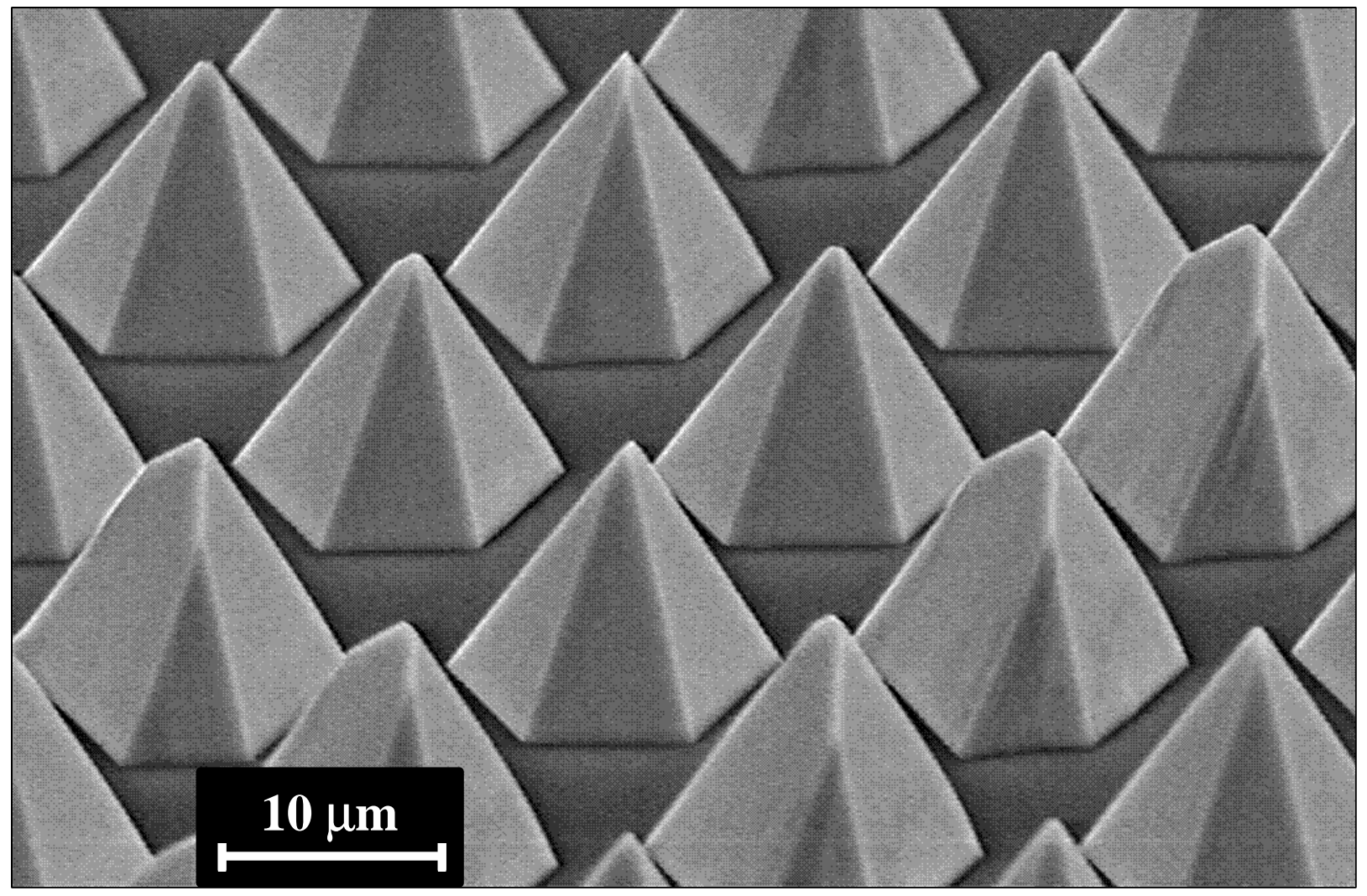

FIG. 1. SEM image of GaN pyramids with a $15 \mu \mathrm{m}$ wide hexagonal base, grown on a (111) Si substrate by selective lateral overgrowth.

The details of the experimental configuration are described elsewhere. ${ }^{10}$ This allowed us to pump, image, and spectrally analyze emission from separate pyramids. 


\section{RESULTS AND DISCUSSION}

The RT emission spectra at different pump densities ( 20-ns-pulses) near the lasing threshold for the 15- $\mu \mathrm{m}$-wide pyramids are shown in Fig. 2. At excitation densities below the lasing threshold, only a spontaneous emission peak with a full width at half maximum (FWHM) of approximately $14 \mathrm{~nm}$ is present. The energy position as well as the spectral width of the peak are very similar to that observed from high-quality single-crystal GaN epilayers. ${ }^{11}$ As the pump density is increased to values slightly above the lasing threshold, a very narrow peak with a FWHM of less than $0.3 \mathrm{~nm}$ appears on the low energy side of the spontaneous emission peak. Note that the FWHM of the stimulated emission peak in high-quality GaN epilayers is typically $2 \mathrm{~nm}$ at RT. The intensity of the peak shown in Fig. 2 increases superlinearly with excitation power. Both drastic narrowing of the spectra and the superlinear increase of intensity suggest that we observe single-mode laser action in the pyramids. The lasing threshold corresponds to an incident

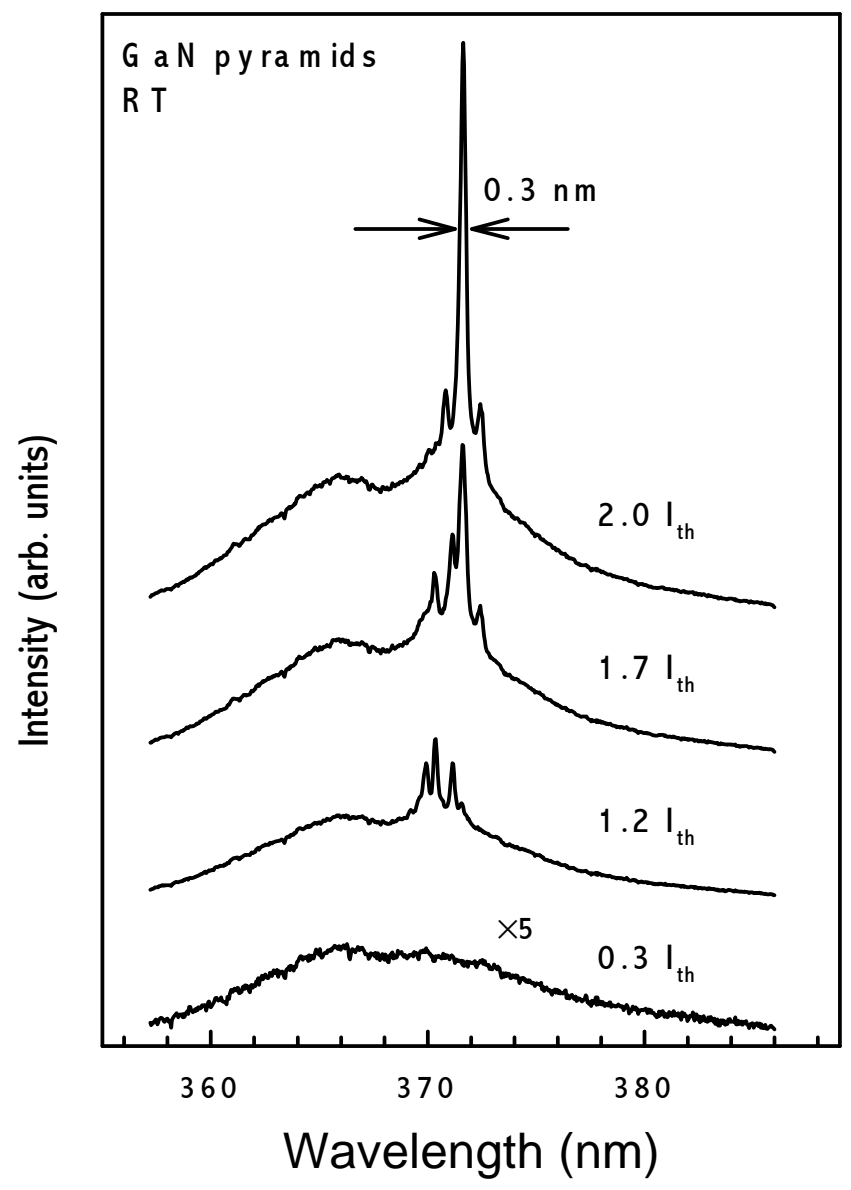

FIG. 2. Emission spectra of a pyramid under different levels of optical pumping above and below the lasing threshold at RT. At pump densities below the lasing threshold only a 14-nm-wide spontaneous emission peak is present whereas at excitation levels above the lasing threshold a narrow single-mode laser peak of less than $0.3 \mathrm{~nm}$ FWHM is observed. 
pump density of several $\mathrm{MW} / \mathrm{cm}^{2}$. However, most of the pump beam scatters off the pyramid surface. In order to estimate the coupling coefficient of the pump beam one has to consider the surface roughness and geometry of each specific pyramid.

For short excitation pulses it was also possible to observe multi-mode laser action in $\mathrm{GaN}$ pyramids, as shown in Fig. 3. In the case of the $15-\mu \mathrm{m}$ wide pyramids (Fig. 3a) the average mode spacing was estimated to be $0.89 \mathrm{~nm}$ which corresponds to the perimeter of $58 \mu \mathrm{m}$ for either a ring or standing-wave cavity (we assume $n_{e}=n-\lambda \cdot d n / d \lambda=2.65$ for the effective refractive index of $\mathrm{GaN}$ at $370 \mathrm{~nm}$, taken from Ref.[12]). For 5- $\mu \mathrm{m}$ wide pyramids (Fig. 3b) the mode spacing increased to approximately $2.2 \mathrm{~nm}$ which corresponds to a $23 \mu \mathrm{m}$ in cavity perimeter. In both cases the perimeter of the cavity appears to be several times larger than the diameter of the pyramid base. This result suggests that the photon flux propagating in the cavity undergoes several $(N \geq 4)$ reflections inside of the pyramid prior to completing one round trip. The difference in mode spacing for the two pyramids could be explained by the difference in their physical dimensions.

To understand the geometry of the cavity formed inside of a pyramid we have to consider the limited penetration depth of the pump. We expect that the gain region can only lie in the vicinity of the surface rather than deep in the body of the pyramid. The existence of a highly

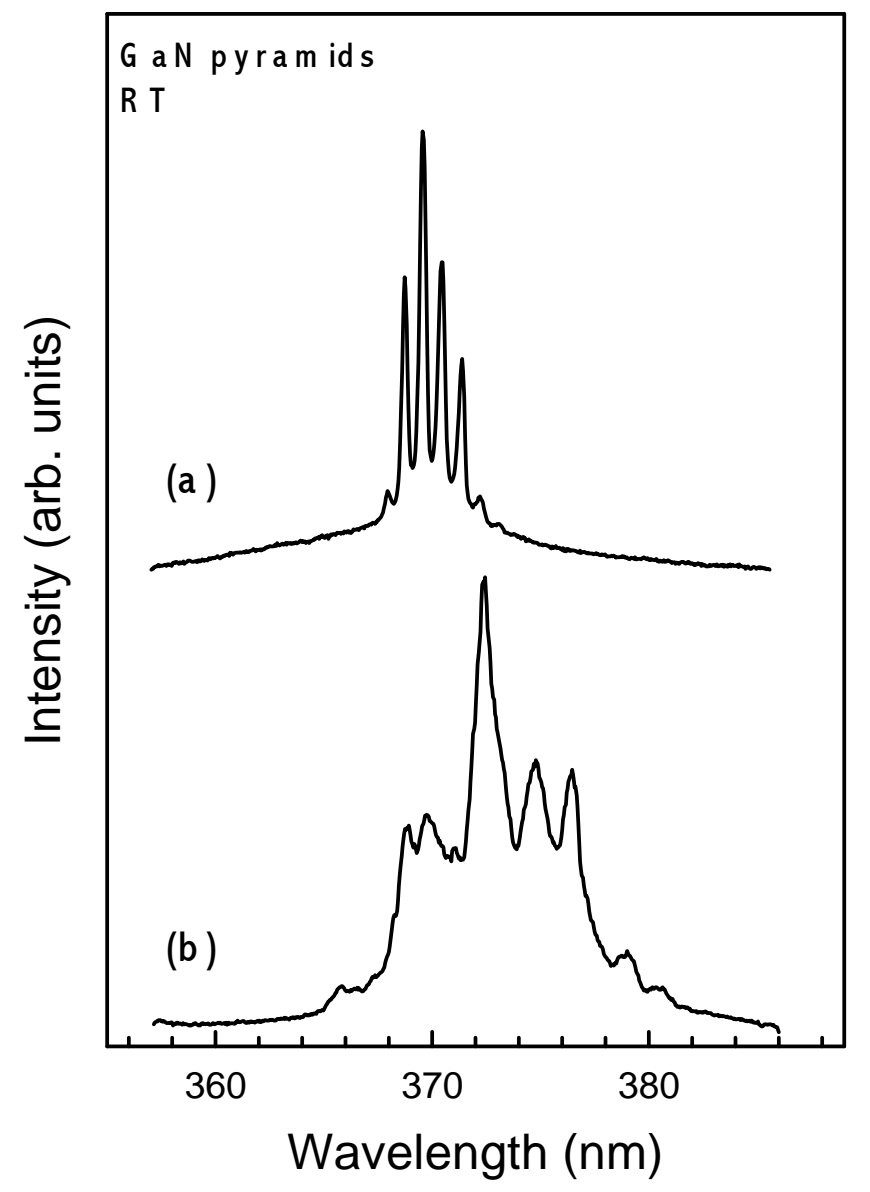

FIG. 3. Multi-mode laser action in (a) $15-\mu \mathrm{m}$-wide and (b) $5-\mu \mathrm{m}$-wide pyramids. The mode spacing corresponds to a cavity perimeter of 58 and $23 \mu \mathrm{m}$, respectively. 
modulated lasing spectrum shown in Fig. 3a with such a short gain region suggests there are very small losses associated with cavity mirror reflectivity and absorption at the lasing wavelength. For multiple reflections, only highly reflective mirrors could provide a reasonable optical feedback. Under normal incidence, a GaN-air interface reflects back approximately $R=0.2$ of the incident signal. Having $N$ near-normal reflections will result only in $R^{N}<10^{-3}$ (for $N \geq 4$ ) of the transmitted signal after one round trip in the cavity, which would require unrealistically high gain in a standing wave cavity with normally oriented end mirrors. Therefore, the cavity inside of the pyramid is most likely of a ring type formed by total internal reflections off the pyramid surfaces. This cavity could potentially lead to a large build-up of the electric field in the pyramid. The collected emission from the sample is believed to be only a scattered fraction of this field, as in the case of scattered stimulated emission observed in GaN epilayers. ${ }^{13}$

We further note that the pyramids used in this work are of much smaller size than conventional edge-emitting LD cavities. Even though efficient carrier injection and coupling of the emission have yet to be developed, these $\mathrm{GaN}$ microstructures could potentially be used as pixel elements and as high density two-dimensional laser arrays. ${ }^{14,15}$ The Si substrate used to grow the pyramids might facilitate the integration of GaN microstructures into Si-based electronics.

\section{CONCLUSION}

We observed single-mode and multi-mode laser action in GaN pyramids under strong optical pumping at room temperature. The pyramids were individually pumped, imaged, and spectrally analyzed through a high magnification optical system using a high density pulsed excitation source. We suggest that the cavity formed in a pyramid is of a ring type, formed by total internal reflections of light off the pyramids' surfaces. The mode spacing of the laser emission was found to be correlated to the size of pyramids. This study suggests that GaN microstructures could potentially be used as pixel elements and high density two-dimensional laser arrays.

\section{ACKNOLEDGEMENT}

The authors would like to thank ONR, BMDO, NSF, and DARPA for the financial support.

\section{REFERENCES}

${ }^{1}$ See, for example, J. J. Song and W. Shan, in Physics and Applications of Group III Nitrides Semiconductor Compounds, edited by B. Gil (Oxford University Press, London, 1998).

${ }^{2}$ S. Bidnyk, T. J. Schmidt, Y. H. Cho, G. H. Gainer, J. J. Song, S. Keller, U. K. Mishra, and S. P. DenBaars, Appl. Phys. Lett. 72, 1623 (1998).

${ }^{3}$ S. Bidnyk, B. D. Little, T. J. Schmidt, J. Krasinski, and J. J. Song, Proc. SPIE 3419, pp. 35-43 (1998).

${ }^{4}$ S. Bidnyk, B. D. Little, T. J. Schmidt, Y. H. Cho, J. Krasinski, J. J. Song, B. Goldenberg, W. G. Perry, M. D. Bremser, and R. F. Davis, to be published in J. Appl. Phys.

${ }^{5}$ S. Nakamura, M Senoh, S Nagahama, N Iwasa, T Yamada, T Matsushita, H Kiyoku, Y 
Sugimoto, T Kozaki, H Umemoto, M. Sano, K Chocho, Jpn. J. Appl. Phys. 37, L309 (1998).

${ }^{6}$ Y. Kato, S. Kitamura, K. Hiramatsu, and N. Sawaki, J. Crystal Growth 144, 133 (1994).

${ }^{7}$ S. Kitamura, K. Hiramatsu, and N. Sawaki, Jpn. J. Appl. Phys. 34, L1184 (1995).

${ }^{8}$ J. J. Song, A. J. Fischer, T. J. Schmidt, S. Bidnyk, and W. Shan, Nonlinear Optics Vol. 18 (2-4), 269 (1997).

${ }^{9}$ W. Yang, S. A. McPherson, Z. Mao, S. McKernan, and C. B. Carter, (unpublished); T. S. Zheleva, O.-H. Nam, M. D. Bremser, and R. F. Davis, Appl. Phys. Lett. 71, 2472 (1997).

${ }^{10}$ S. Bidnyk, B. D. Little, Y. H. Cho, J. Krasinski, J. J. Song, W. Yang, and S. A. McPherson, Appl. Phys. Lett. 73, 2242 (1998).

${ }^{11}$ X. H. Yang, T. J. Schmidt, W. Shan, J. J. Song, and B. Goldenberg, Appl Phys. Lett. 66, 1 (1995).

${ }^{12}$ E. Ejder, Phys. Status Solidi A 6, 445 (1971).

${ }^{13}$ S. Bidnyk, T. J. Schmidt, G. H. Park, and J. J. Song, Appl. Phys. Lett. 71, 729 (1997).

${ }^{14}$ K. Iga, S. Kinoshita, and F. Koyama, Electron. Lett. 23, 134 (1987).

${ }^{15}$ F. Koyama, K. Tomomatsu, and K. Iga, Appl. Phys. Lett. 52, 528 (1988). 